\title{
International Conference on Chlamydial and Mycoplasma Human Infections
}

19-20 April, 2007, Ferrara, Italy

\author{
Carlo Contini \\ Section of Infectious Diseases, Department of Clinical \& Experimental Medicine, \\ University of Ferrara, 44100 Ferrara, Italy
}

Tel.: +39532 455 490; Fax: +39 532237 707; cnc@unife.it

The International Conference on Chlamydial and Mycoplasma Human Infections was held in Ferrara, Italy during April 19-20, 2007.

With nearly 20 countries represented, this conference attracted more than 200 participants, including physicians, clinical microbiologists, researchers, trainees and other professional groups, thus providing a unique global networking and educational opportunity.

According to the program leaflet, the international meeting was especially addressed to scientists and physicians involved in clinical laboratory research and drug treatment of chlamydia and Mycoplasma infections. The main topics of the conference were epidemiology, pathogenesis, clinical aspects, diagnostic advances, treatment and vaccine trial perspectives of chlamydia and $\mathrm{Myco-}$ plasma human infections. Diseases of particular interest, including atherosclerosis, multiple sclerosis (MS), chronic fatigue syndrome (CFS) and ocular lymphomas, in whom the role of these pathogens seems to be called in cause, were particularly stressed. Besides the regular talks, oral and poster presentations dealing with most conference topics have stimulated the discussion in both sections of this article, chlamydia and Mycoplasma.

Famous speakers at the conference came from academic and research institutions and included Pekka Saikku (Houlu University, Houlu, Finland), Charles Stratton (Vanderbilt University Medical Center, Nashville, USA), David Taylor Robinson, (Imperial College, London, UK), Ken Waites (University of Alabama, Birmingham, USA), Agatha Subtil (Pasteur Institute, Paris, France), Alain Blanchard (University of Bordeaux, Villenave D' Ornon, France) and Cecile Bebear (University of Bordeaux, Bordeaux, France).

The Federation of European Microbiology Society (FEMS) was the main sponsor of the conference. Other sponsors including Pfizer, Roche, GlaxoSmithKline (GSK), Copan and Eurospital Italia have supported this international workshop. The conference was under the auspices of a number of scientific Italian societies, including the Italian Society of Infectious and Tropical Diseases (SIMIT), Italian Society of Neurology (SIN), Italian Society of Ophthalmology (SOI), Italian Society of Respiratory Medicine (SIMER), Italian Society of Internal Medicine (SIMI), Italian Society of Rheumatology (SIR) and Italian Society of Obstetrics and Gynecology (SIGO).

\section{Chlamydia}

After the introducing overview held by Carlo Contini, chairman of the conference, the section on chlamydia began with the lecture of Agatha Subtil (Pasteur Institute, Paris, France). She focused on chlamydia entry into the cell, how it escapes host defenses and replication in a unique intracellular organelle. The possible strategies of control that this pathogen makes use of to avoid human defenses were presented and discussed. Her very interesting studies have underlined the endocytosis mechanism that is clathrin-mediated and occurs during chlamydial infection. The use of different strategies, either on the basis of genomic data or not, is a particular target for the design of potential vaccines against infections. Subtil explained that to be effective, such vaccines are likely to require the incorporation of several antigens, able to stimulate multiple arms of the immune system.

Meja Leinonen (National Public Health Institute, Houlu University, Finland), has found that certain HLA haplotype markers correlate with serological markers of Chlamydia pneumoniae infection in patients with acute coronary syndrome. A multiple logistic regression analysis showed that $H L A-B 35$ allele is the strongest risk gene for the combined serological markers of C. pneumoniae. Being male and smoking may further strengthen the association between $H L A-B 35$ and markers of $C$. pneumoniae infection. Interestingly, HLA-B35 is also associated with cardiovascular risk in Finnish patients. In this regard, another representative scientist, Pekka Saikku, emeritus professor of microbiology (Houlu University) was the first scientist to discover a link between C.pneumoniae and atherosclerosis. There is much circumstantial evidence suggesting that $C$. pneumoniae may play a role in atherosclerosis and in an alarmingly large number of other chronic diseases, but proof, owing to the nature of the problem, is hard to come by. Saikku explained that in order to prevent the persistence of the agent and the development of atherosclerosis, this chronic disease should be treated as early as possible. Chronic C. pneumoniae infection is not possible to eradicate, at least with antibiotic monotherapy. A clear indication of this was supported by the fact that treatments did not have an effect on markers of chlamydial infection. Saikku also focused on the need to develop combinations of drugs, which, as in tuberculosis and helicobacter infections, are able to 
eradicate the pathogen. Statins, aspirin and dietary polyphenolic compounds are among them.

Charles Stratton (Department of Pathology, Vanderbilt University Medical Center, Nashville, TN, USA), spoke about the relationship between C. pneumoniae and MS, which was discovered in 1999 by Subramaniam Sriram (MS Center, Vanderbilt Stallworth Rehabilitation Hospital, Nashville, TN, USA). $\mathrm{He}$ undertook immunohistochemical and molecular and ultrastructural studies on CNS tissues, which demonstrated C.pneumoniae in ventricular wall and subependimal regions in subsets of MS patients but not in control individuals with other neurological diseases. These results provide further evidence of the strong implication of C.pneumoniae in this disease. Stratton also focused on the need to develop combinations of drugs to be employed in MS clinical trials. He stated that conventional antimicrobial therapy may not kill the intracellular organism, but rather induce it to undergo a phenotypic shift to a persistent cryptic form that resists treatment and microenvironmental conditions [1]. This form has not been fully explored yet, but analogies with other intracellular pathogens able to survive under stress conditions suggest that it may be anaerobic. In this setting, the search of drugs that can kill the cryptic form would be effective.

Henning Zeidler (Division of Rheumatology, University of Hannover, Germany) gave novel insights into the diagnosis of associated chlamydia and arthtritis. He noticed that the commercial arrays, including reverse transcriptase-PCR are not sensitive enough with regard to intra-articular chlamydial detection; the best results are obtained with in-house PCR systems but sensitivity depends on the extraction method. Zeidler underlined the need for commercially available, highly sensitive assays, which allow valid interlaboratory comparisons and use in clinical diagnosis.

Problems of a similar extent concerning the general approach to serological diagnostic infection of C. pneumoniae were discussed by Corinna Hermann
(Institute of Biochemical Pharmacology, University of Konstanz, Germany). She reported the latest advances on chlamydial proteinomics and presented a number of experiments performed with highly purified and immunoreactive proteins of C. pneumoniae for a more sensitive serological approach. Concerning chlamydial persistence, she also indicated that in vivo Toll-like receptor (TLR) 2 and TLR4 are not important for the development of antibodies and elimination of $C$. pneumoniae.

The fact that Chlamydia trachomatis is responsible for 82-92 million new cases of $C$. trachomatis genital tract infections each year, and that this trend in Europe continues to rise alarmingly, was reported by Roberto Cevenini (Microbiology, University of Bologna, Italy). Sexually transmitted infections caused by $C$. trachomatis are considered the 'silent disease' because infection rarely produces symptoms, especially in women. Left untreated, Chlamydia can trigger pelvic inflammation leading to chronic disease and infertility. C. trachomatis is also strongly implicated in the transmission of HIV and increases the probability of introducing high-risk human papillomavirus genotypes that induce cervical intra-epithelial neoplasia. For these reasons, public health authorities in many developed countries have launched chlamydia control programs, even if in some trials vaccination predisposed to more severe disease.

Switching from C.trachomatis to Chlamydia psittaci, this pathogen was proposed as a potential cause of lymphoma of the ocular adnexa (OAL). This was explored by the pathologist Andreas Ferreri (S. Raffaele Hospital, Milan, Italy). OAL share some clinicalpathologic features with gastric lymphomas, for which a pathogenic link with Helicobacter pylori-related follicular gastritis was previously established [2]. He noticed that $C$. psittaci infection was evident in more than $80 \%$ of subjects with OAL; in addition, a strong amplification DNA signal was found in peripheral blood mononuclear cells (PBMCs) from approximately $40 \%$ of OALs. Ferreri and his coworkers also evaluated the efficacy of doxycycline against $C$. psittaci in patients with OAL. Of interest, PBMC DNA was not detected after 1 month of doxycycline treatment in seven investigated patients.

\section{Mycoplasma}

Human Mycoplasma infections were explored by David Taylor-Robinson, (Imperial College, London, UK). His lecture, chaired by Shmuel Razin (The Hebrew University-Hadassah Medical School, Jerusalem, Israel) and Dimitri Kafetzis (University of Athens, Athens, Greece), was one of the topics that gave rise to most expectation in the conference. Taylor-Robinson presented all the major topics concerning human mycoplasmas. He also gave information regarding the distribution and significance of Mycoplasma penetrans, Mycoplasma pirum and Mycoplasma spermatophilum. Mycoplasma amphoriforme is a newly described species isolated from patients with chronic bronchitis, primarily those with B-cell deficiencies. However, its clinical importance in the general population remains to be determined. He raised the important questions of whether there is a link between tubal factor infertility and Mycoplasma hominis, calculi and ureaplamas, as well as pyelonefritis and $M$. hominis. With concern to joint diseases, Taylor-Robinson notified that Mycoplasma fermentans has been found by PCR technology in the joints of more than one fifth of patients with rheumatoid arthritis and other chronic inflammatory disorders. Special mention was also given to Mycoplasma genitalium, which has been detected in patients with sexually acquired reactive arthritis, thus suggesting that arthritis of mycoplasmal etiology should be considered in patients with hypogammaglobulinemia who develop an abacterial septic arthritis. Another crucial point he touched upon, is the knowledge status between $M$. fermentans, or other mycoplasmas, and the development of HIV infection.

Mycoplasma genomics is currently undergoing a revolution, boosted by its interaction with informatics. Alain Blanchard (Institut de Biologie Végétale 
Moléculaire, University of Bordeaux, Villenave D' Ornon, France), has developed a database, named MolliGen [101], dedicated to the comparative genomics of Mollicutes. Mycoplasmas are among the most evolved bacteria, a feature that they share with other organisms, including insect endosymbionts. In particular, the research group of Blanchard has recently developed a typing assay based on a multilocus variable-number of tandemrepeats analysis that provides a better discriminatory power compared with other previously described approaches [3]. This system is a powerful typing method that benefits from genomics and has potential for most mycoplasmas, including those infecting humans. Mycoplasmas, reported Blanchard, cannot be considered as an evolution dead-end because they retain the ability to gain new genetic material, probably for keeping their competitive edge over their hosts. Interestingly, the density of repeats is variable among Mycoplasma genomes, suggesting that the means to generate genetic variation can differ from one species to another. These regions are important, not only because they help us to better understand genome rearrangements among strains, but also because they can be used as targets to develop typing assays for epidemiological purposes.

The field of ureaplasmas and related diseases was exhaustively explored by Ken Waites (Diagnostic Mycoplasma Laboratory, University of Alabama, Birmingham, AL, USA). He strengthened the mechanisms involved in pathogenesis, as well as most clinical and diagnostic aspects of Ureaplasma spp. He focused on the fact that Ureaplasma spp. should be considered as causes of pneumonia, bacteremia and meningitis in neonates, especially in the absence of other pathogens. Waites underlined that effective antibiotic trials are needed to determine whether this can lessen inflammation, bronchopulmonary dysplasia and improve long-term outcomes.

An important aspect related to clinics of Mycoplasma was presented by Jo Nijs (Division of Musculoskeletal Physiotherapy, Department of Human Physiology, Vrije Universiteit, Belgium).
People with CFS suffer from multiple symptoms that include fatigue, problems with memory, concentration and sleep, and musculoskeletal pain. The association of any one infectious agent with CFS has been difficult to establish and is complicated further by the lack of a known lesion or diseased tissue to sample. In European CFS patients, Nijs noticed that $M$. hominis infections were more common compared with $M$. pneumoniae and $M$. fermentans infections. By contrast, in American CFS patients there is a slightly different pattern of mycoplasmal infection, in that $M$. pneumoniae is more prevalent than $M$. hominis and M. penetrans. Nijs found that CFS patients might undergo deleterious consequences on bone density. M. fermentans, which has been frequently associated with CFS, produces a lipopeptide named 2-kDa macrophage-activating lipopeptide, which has been shown to enhance bone resorption in part by stimulating the formation of prostaglandins. Special mention was also given to the relationship between $\mathrm{Myco-}$ plasma and fibromyalgia. A high prevalence (50-70\%) of multiple Mycoplasma $s p$. was found in patients with fibromyalgia, which showed very interesting immunophenotyping profiles.

With regards to a CFS setting, an interesting presentation was given by Delia Racciatti from the Clinic of Infectious Diseases of University of Chieti, Italy. She stated that sexual mycoplasmal infections are more frequently reported in women with CFS than in men, and correlated with fatigue, joint pain and age.

Regarding the possible role of $\mathrm{Myco}$ plasma in MS, Edward L. Hogan (Institute of Molecular Medicine and Genetics [IMMAG], University of Galway, Ireland) also held an excellent presentation. He demonstrated that the cross-reactivity of antibodies specific for the acetyl-cerebroside structure with purified M. fermentans glycoglycerolipid (MfGL-II), indicates molecular mimicry between myelin and microbial acyl-glycosides, and raises the possibility that human infection with Mycoplasma induces autoimmune demyelination perhaps by elicitation of innate immune responses.
Other hot topics at the conference related to the putative role of mycoplasmas, as well as of Brucella Spp., Borrelia spp. and C. pneumoniae in patients with CFS, Gulf war syndrome and fibromyalgia. These findings were presented by Garth Nicolson (Institute of Molecular Medicine, Huntington Beach, CA, USA), who highlighted that there was no correlation between the type of coinfection and severity of signs and symptoms. Large subsets of CFS patients show evidence of bacterial and/or viral infection(s), and these infections may contribute to the severity of signs and symptoms found in these patients. Moreover, the incidence of C. pneumoniae or human herpesvirus (HHV)-6 was similar in Mycoplasmapositive and -negative patients, and the converse was also found in active HHV-6-positive and -negative patients.

The questions concerning the difficulties associated with Mycoplasma serology-based diagnosis were raised by Zvi Grenberg (Research and Development of Savyon Diagnostics, Ashdod, Israel). Serology is still the method of choice, particularly for screening purposes. However, it will only be a useful tool if we know how to use it properly.

Cecile Bebear from the University of Bordeaux is involved in an important international multicenter study for the Mycoplasma susceptibility subcommittee coordinated by Ken Waites. She discussed the progress made in antimicrobial therapy (ATB) against mycoplasmas, and resistance. The question of whether it is necessary to test ATB susceptibility in mycoplasmas is especially true for M. hominis and Ureaplasma spp. in lower genital specimens when estimated to be responsible for infection and in immunosuppressed patients. For $M$. pneumoniae, ATB susceptibility would eventually be carried out in therapeutic failure, or for epidemiological studies.

\section{Conclusion}

One of the most attractive presentations for both clinicians and microbiologists regarded the role of $C$. pneumoniae and Mycoplasma in chronic diseases. 
M. pneumoniae is a major cause of community-acquired respiratory illness in children and adults, both in terms of clinical severity and numbers affected. A consistent body of evidence supported the notion that the organisms may remain viable long after an acute respiratory infection has subsided. The mechanisms of extrapulmonary manifestations (neurologic, dermatologic, cardiologic, rheumatologic and hematologic) appear to include disseminated infection (especially in patients with humoral immunodeficiencies); autoimmune phenomena, and, possibly, toxin production. However, the exact mechanism of invasion of extrapulmonary tissues, such as the CNS, and the late-onset symptoms after several days to a few weeks, remains unknown.

In my opinion, clinicians should be aware of their potential role when confronted with symptoms of unknown

\section{Bibliography}

1. Stratton CW, Wheldon DB:

Multiple sclerosis: an infectious syndrome involving Chlamydophila pneumoniae. Trends Microbiol. 14(11), 474-479 (2006).

2. Ferreri AJ, Ponzoni M, Viale E et al.: Association between Helicobacter pylori infection and MALT-type lymphoma of the ocular adnexa: clinical and therapeutic implications. Hematol. Oncol. 24(1), 33-37 (2006). cause, especially if the patient's history does not include previous symptoms from the respiratory tract.

The most important current question regarding C.pneumoniae pathogenesis is: what is the pathologic significance of the organism in atheroma, the lungs, cerebrospinal fluid and other tissues? The consequence of persistent infection is yet to be explored. C.pneumoniae research suggests that it can enter the walls of various blood vessels via circulating monocytes, and remain for years, spreading and establishing chronic infections, which trigger the inflammation that causes heart attacks, stroke and probably vasculitis and myocarditis. However, it must be questioned whether C.pneumoniae is present in any great number and therefore, whether it is of pathogenic importance. An innocent bystander at the scene of the crime, as powerful

3. Barre A, de Daruvar A, Blanchard A:

MolliGen, a database dedicated to the comparative genomics of Mollicutes. Nucleic Acids Res. 1, 32(Database issue), D307-D310 (2004).

\section{Website}

101. Molligen http://cbi.labri.fr/outils/molligen/L molecular techniques have demonstrated, or a pathogen which plays an active role? Further studies to establish causality are urgently needed. Further research is also required to verify the test material of choice to detect C. pneumoniae and draw conclusions about the safety and benefit of prolonged antibiotic use to eradicate the pathogen.

Efforts to standardize methods are worthy, but the real problem is that current methods to detect chronic infection need a wider deepening from the molecular and genetic point of view. Therefore, the size of any potential public health problem remains in doubt.

\section{Financial disclosure}

The authors have no relevant financial interests including employment, consultancies, honoraria, stock ownership or options, expert testimony, grants or patents received or pending, or royalties related to this manuscript.

\section{Affiliation}

- Carlo Contini Section of Infectious Diseases, Department of Clinical \& Experimental Medicine, University of Ferrara, 44100 Ferrara, Italy

Tel.: +39532291310;

Fax: +39532 237707 ;

cnc@unife.it 